\title{
Gasbocca im Zentralkrater des Aetna
}

Das nachfolgende Farbbild, aufgenommen von Tomáš Míček und vom Verlag Kümmerly \& Frey aus dem Bildband «Sizilien» der "Geographica Helvetica» in verdankenswerter Weise zur Verfügung gestellt, gibt einen Anblick wieder, der sich anläßlich der AetnaEruption vom Herbst 1968 an der Terrasse des Zentralkraters bot. Aus der Tiefe einer «Bocca», einer rundlichen Öffnung, die im Kraterfeld aufgesprengt worden ist, leuchtet die Glut hochstehender Lava. Die wirksamen Kräfte reichen zwar nicht aus, die brodelnde Masse ausfließen zu lassen. Doch geht eine intensive Entgasung vor sich. Sie führt dazu, daß in Intervallen von etwa fünf Minuten heftige Explosionen erfolgen. In weitem Umkreis entweichen die vulkanischen Gase auch durch Erdspalten, und da sie einen hohen Anteil an Wasserdampf enthalten, der beim Austreten infolge Kondensation sichtbar wird, legen sich weithin breite Schwaden über den Boden. Gleichzeitig bewirkt die rasche Abkühlung der heißen Exhalationen eine Sublimation von mineralischen Bestandteilen, vorab von Schwefel. So erscheint die Umgebung der Austrittsstelle mit hellen Flecken gesprenkelt, die sich deutlich vom dunklen Grund abheben.

Das Bild hält einen augenblicklichen Zustand im Gang einer Aetna-Eruption fest, eine vorübergehende Erscheinung am Rande des eigentlichen Geschehens. Die Hauptaktivität äußerte sich am Hang des Vulkankegels, wo Lateralkrater Lockermassen und Lava förderten. Im Zentralkrater aber traten nur noch vulkanische Gase zutage. Bei der dargestellten Öffnung handelt es sich um ein «Blowhole», eine Art von vorübergehend geöffnetem Ventil, durch welches hochgespannte Gase, die das Innere des Vulkans durchsetzen, einen Ausweg ins Freie finden. Als nach etwa zwei Jahren die Eruption abzuklingen begann, stellte auch die «Bocca soffiante», wie dieses Blowhole genannt wurde, ihre Tätigkeit ein. Ringsum gab der Boden nach und sank in die Tiefe. Wo einst der Feuerschlund sich geöffnet hatte, gähnt jetzt ein schroffer Abgrund, dessen Wände von den weißen Dampfschwaden einer starken Fumarolentätigkeit belebt werden.

In der Erforschung der vulkanischen Erscheinungen, der in der Geologie eine immer stärkere Bedeutung zukommt, nimmt der Aetna dank seiner leichten $\mathrm{Zu}$ gänglichkeit und seiner fortdauernden Tätigkeit eine Schlüsselstellung ein. Die Beobachtung der jüngsten Eruptionen hat die Beweise verdichtet, daß tektonische
Kräfte in Ostsizilien die Erdkruste in etwa nord-südlicher Richtung dehnen. Nachweisbar rissen die ersten Zerrspalten in der Triaszeit den Grund eines seichten Meeres auf. Dabei ergossen sich basaltische Magmamassen in der Gegend des heutigen Ragusa über Meeresboden. Ähnliche submarine Ausbrüche erfolgten in der Jura- und in der Kreidezeit. Weiter im Norden, im Gebiet der Monti Iblei, breiteten sich im Tertiär submarine Lavaströme aus. Vor etwa 500000 Jahren dürfte die Zerspaltung der Erdkruste das Gebiet des heutigen Aetnas erreicht haben. Basaltmagmen drangen in tonige Meeresablagerungen ein und durchbrachen sie an vielen Stellen, wobei sich der Unterschied zwischen submarinen und subaerischen Laven deutlich manifestiert. Marine Sedimente des mittleren Quartärs stehen heute am Osthang des Aetna in einer Höhe von 800 Metern ü. M. an, am Aspromonte in Kalabrien gar in 1400 Metern. Mit diesen beträchtlichen Hebungen ging ein grabenbruchartiges Absinken des Meeresbodens zwischen Sizilien und Kalabrien einher. Im Bereiche des Aetna durchkreuzen sich darum das von West nach Ost verlaufende System der erwähnten Zerrspalten mit einer Serie von küstenparallelen Staffelbrüchen. Auf diese Weise ist die Erdkruste unter dem Vulkan in ein Schollenmosaik aufgelöst worden. Hier fand der Magmaaufstieg wenig Widerstand, und es entstanden im Laufe der Zeit zahlreiche Kleinvulkane, die vom Hauptschlot weitgehend unabhängig waren. Mit der Ausweitung des Bruchfeldes nach Westen griffen auch die Eruptionen in diese Richtung aus, und in diesem Sinne verlagerte sich ebenfalls der Zentralkrater. Die Anordnung der Ausbruchsöffnungen an den Flanken des Aetna und in seiner unmittelbaren Nachbarschaft spiegelt die Bruchtektonik des Untergrundes deutlich wider. Infolge der tektonischen Vorgänge, die auch heute noch fortdauern, erscheint der Aufbau des Aetna außerordentlich verwickelt. Es handelt sich nicht um einen einfachen Vulkan, sondern um ein vielgestaltiges Gebirge, das aus mehreren großen und unzähligen kleinen Vulkanen zusammengewachsen ist. Seine Erforschung gestaltet sich deshalb sehr schwierig. Die in den letzten Jahren mit modernen petrographischen, geophysikalischen und geochemischen Methoden durchgeführten Untersuchungen haben schon viel-

Prof. Dr. Fritz Bachmann, Mörlistrasse 23, 8006 Zürich 
versprechende Ergebnisse gezeitigt. Diese werfen neues Licht auf den Mechanismus der Eruptionen und vor allem auch auf immer engere Zusammenhänge zwischen Vulkanismus und Geotektonik. Am eingehendsten konnte bisher die Eruption im Jahre 1971 erfaßt werden. Von Anfang an waren die Vulkanologen zur Stelle. Es wurde nicht nur der Ablauf der Ereignisse bis in alle Einzelheiten beobachtet, sondern auch während allen Ausbruchsphasen im Internationalen Institut für Vulkanologie in Catania eine gründliche Analyse der geförderten Lava vorgenommen. Das Volumen der ausgeflossenen Lava betrug rund 75 Millionen Kubikmeter, was etwa einer Masse von 200 Tonnen entspricht. Die Eruption ist nach diesen Angaben als verhältnismäßig bescheiden zu beurteilen, förderte doch der Ausbruch des Jahres 1669 beispielsweise mindestens eine zehnmal größere Menge. Doch erwies sich dieser jüngste Ausbruch in wissenschaftlicher Hinsicht als äußerst aufschlußreich. Er wich weitgehend von den bisherigen Erfahrungsregeln ab und ließ sich kaum mit bereits bekannten Eruptionen vergleichen. Doch die Kenntnis der Vorgeschichte und des Ablaufs sowie die Ergebnisse zahlreicher Analysen vermochten den Ausbruchsmechanismus hinreichend zu erklären. Es gelang u. a. der Nachweis, daß die im Innern des Berges klaffende Spalte, in welche das Magma eingedrungen war, schon seit langem bestanden hatte und ihre Entstehung einer tektonischen Zerrung verdankt. Bereits beim Ausbruch von 1928 war Magma hier eingedrungen und in den unteren Partien der Spalte zu festem Gestein erstarrt. Als dann 1971 im oberen offenen Teil neue Magmamassen nach Osten flossen, stießen sie auf den verstopften Teil und suchten sich hangabwärts zwischen den Schichten des Vulkans einen neuen Weg. Schließlich traten sie 500 Meter tiefer zutage, wobei durch einen Lavastrom im Laufe des Aprils das Observatorium und die obere Station der Schwebebahn zerstört wurden.

Nach A. Rittmann

\section{Buchbesprechung}

Míček Tomáš und andere Autoren, «Sizilien-Liparische Inseln», Kümmerly \& Frey, Geographischer Verlag, Bern, 1973. Fr. 48.-

Der Bildband hält sich nicht an herkömmliche Klischees der Insel, ohne die - nach J. W. Goethe - Italien kein Bild in der Seele mache, weil hier der Schlüssel zu allem liege. Nicht die bunte Vielfalt des kulturellen Niederschlags, den die Jahrhunderte währende, immer wieder wechselnde Fremdherrschaft auf Sizilien hinterlassen hat, steht im Vordergrund, sondern das Zusammenwirken von geographischen, ethnographischen, archäologischen und historischen sowie aber auch geologischen Realitäten, welches letztlich die Gesamtheit der Mittelmeerinsel ausmacht. Auch werden die Liparischen Inseln in den sizilianischen Raum einbezogen, die zwar außer archäologischen kaum nennenswerte Kulturdenkmäler aufweisen, in ihrer Landschaft aber und in der Lebensweise ihrer Bevölkerung eng mit Sizilien verwandt sind. Aufnahmen aus dem Innern Siziliens vermitteln Eindrücke von noch unverfälscht erscheinenden mediterranen Landschaften. Vor allem aber ist auf den informativen Gehalt der Textbeiträge hinzuweisen. Einer ansprechenden pflanzengeographischen Übersicht folgt ein volkskundliches Kapitel, das sich mit verschiedenen Lebensbereichen, manchen besonderen Erscheinungen und einigen ausgewählten Örtlichkeiten befaßt. Vom mittelalterlichen und modernen Sizilien werden nur einige wesentliche Aspekte behandelt. Eingehender befaßt sich dann ein nächster Bericht mit den archäologischen Forschungsergebnissen und den ur- und frühgeschichtlichen Verhältnissen. Der verbleibende Raum ist den vulkanischen Erscheinungen in Ostsizilien und im Gebiet der Liparischen Inseln gewidmet, vorab dem Aetna und seinen Eruptionen. So darf der prachtvoll ausgestattete Bildband zugleich als wertvolle länderkundliche Ergänzung gelten, die manche Lücke zu schließen vermag.

Fritz Bachmann 